\title{
Rigorous mode solver for multilayer cylindrical waveguide structures using constraints optimization
}

\author{
Uwe Langbein • Udo Trutschel • Andreas Unger • \\ Michel Duguay
}

Received: 28 July 2009 / Accepted: 22 October 2009 / Published online: 14 November 2009

(C) Springer Science+Business Media, LLC. 2009

\begin{abstract}
A rigorous mode solver for multilayer fiber configurations using a Constraint Optimization method is presented. For an arbitrary number of dielectric or metallic layers the cylindrical wave equations are solved exactly. The transition between the different layers implies a coupling of all field components. Case examples of three unconventional fiber structures (Air-ARROW-fiber, metal-coated fiber, core to ring fiber coupler) are presented. Mode dispersions charts and field characteristics are calculated to demonstrate the performance of the constraints optimization method applied.
\end{abstract}

Keywords Fiber mode solver · Contraints optimization · ARROW fiber ·

Metal cladded fiber $\cdot$ Plasmons

\section{Introduction}

Based on the recent progress in integrated optic and fiber technologies, we can distinguish between two basic principles of light guiding. First, in conventional optical fibers, light guiding is achieved by total internal reflection (Born and Wolf 1959; Okoshi 1982; Boerner

\author{
U. Langbein \\ University of Applied Sciences, Wiesbaden, Germany \\ U. Trutschel $(\varangle) \cdot$ A. Unger \\ Design \& Optimization of Optical Systems (DOOS), Tabarz, Germany \\ e-mail: utrutschel@doos.de \\ U. Trutschel \\ Institute for System Analysis \& Applied Numerics (ISAAN), Tabarz, Germany
}

A. Unger

Max Planck Institute for Polymer Research, Mainz, Germany

M. Duguay

Laval University, Quebec, Canada 
and Trommer 1989; Bures 2009). Second, in the so-called ARROW and Bragg fibers, light guiding takes place utilizing multi wave interference effects similar to Fabry-Perot cavities (Yeh et al. 1978; Yan and Shum 2004; Rowland et al. 2008). It is still believed in the optical research community that the analysis of ARROW and Bragg fibers is more challenging than the analysis of conventional fibers. The here proposed approach allows the analysis of multilayer cylindrical waveguide structures in general by identifying the modal field excitations supported by the corresponding waveguide. The light guiding mechanism does not matter at all. In particular, absorbing layers such as metal layers can be included into the analysis.

In this paper the involved eigenvalue problem of mode finding in multilayer cylindrical waveguide structures is redefined as a minimization problem with constraints. The constraints optimization method has a long history in the field of operation research and was developed for multi-parameter optimization in complex decision problems. The basic foundation of this theory was already laid by Danzig and coworker in 1947 (Danzig 1963; Luenberger 1984). The application of this old optimization method to the modern field of fiber optics turned out to be quite successful. We will show below that the eigenvalue problem for the determination of fiber modes can be reformulated as minimization problem with constraints. The applied method avoids local minima of the eigen value equation restricting the search space by means of constraints. In this way modal fields characterized by complex-valued propagation constants and complex-valued field components can be determined.

\section{Algorithm}

The algorithm presented here has a modular structure, i.e. the optical properties of each homogeneous cylindrical layer within the total waveguide are condensed into an operational unit. Thus the complete waveguide can be described by an appropriate succession of operational units, denoting the successive application of unit by unit. In this respect, our algorithm shows some similarities with the well known matrix formalism for planar optical layer stacks (Chilwell et al. 1984)

Transferring the Maxwell's equations to cylindrical coordinates, we end up with the electric field vector $\mathbf{E}=\left[\mathrm{E}_{\mathrm{r}} \mathrm{E}_{\phi} \mathrm{E}_{\mathrm{Z}}\right]$ and the magnetic field vector $\mathbf{B}=\left[\mathrm{B}_{\mathrm{r}} \mathrm{B}_{\phi} \mathrm{B}_{\mathrm{Z}}\right]$. The modes propagate in $z$-direction with the (complex-valued) propagation constant ' $\beta$ ' (Fig. 1). There are four transverse and two longitunal electric and magnetic field components.
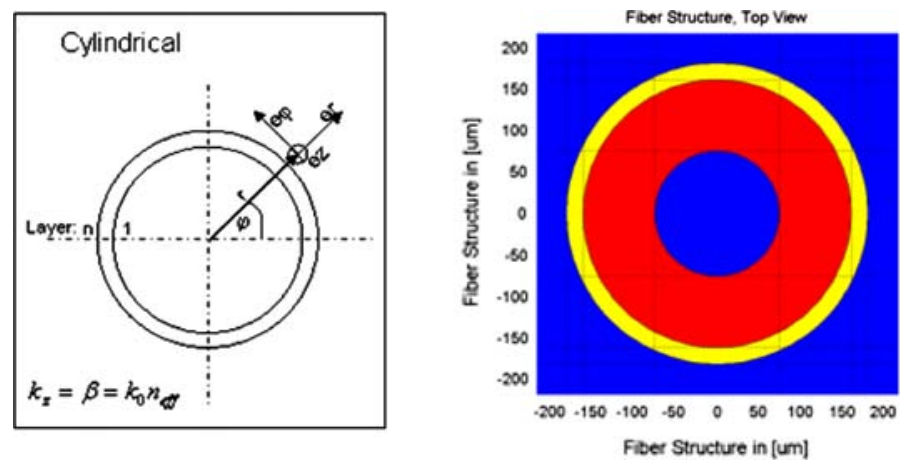

Fig. 1 Cylindrical coordinates (left) and multilayer fiber structure (right) each homogeneous layer shall be characterized by a complex-valued dielectric function $\varepsilon_{\mathrm{j}}$ 
As long as the optical field pattern within a given homogeneous cylindrical layer, specified by a dielectric function ' $\varepsilon_{\mathrm{j}}$ ', is considered, it will be sufficient to solve the wave equation for the longitudinal field components $E_{Z}^{j}$ and $B_{Z}^{j}$ only, for the transverse electric and magnetic field components can be expressed in terms of their longitudinal counterparts, see Eq. 1 .

$$
\begin{aligned}
B_{r}^{j}(r, \varphi) & =\frac{i}{k_{0}^{2} K_{j}^{2}}\left\{k_{0} \beta\left[\frac{\partial}{\partial r} B_{Z}^{j}(r)\right]-i v \frac{k_{0}^{2} \varepsilon_{j}}{r}\left[E_{Z}^{j}(r)\right]\right\} e^{i \nu \varphi} \\
B_{\phi}^{j}(r, \varphi) & =\frac{i}{k_{0}^{2} K_{j}^{2}}\left\{i \nu \frac{k_{0} \beta}{r}\left[B_{Z}^{j}(r)\right]+k_{0}^{2} \varepsilon_{j}\left[\frac{\partial}{\partial r} E_{Z}^{j}(r)\right]\right\} e^{i \nu \varphi} \\
E_{r}^{j}(r, \varphi) & =\frac{i}{k_{0}^{2} K_{j}^{2}}\left\{k_{0} \beta\left[\frac{\partial}{\partial r} E_{Z}^{j}(r)\right]+\frac{i \nu}{r}\left[B_{Z}^{j}(r)\right]\right\} e^{i \nu \varphi} \\
E_{\phi}^{j}(r, \varphi) & =\frac{i}{k_{0}^{2} K_{j}^{2}}\left\{\frac{i \nu k_{0} \beta}{r}\left[E_{Z}^{j}(r)\right]-\left[\frac{\partial}{\partial r} B_{Z}^{j}(r)\right]\right\} e^{i \nu \varphi} \\
k_{0}=\frac{2 \pi}{\lambda} ; \quad K_{j}^{2} & =\varepsilon_{j}-\beta^{2} ; \quad \lambda: \text { Wavelength; } \quad \nu: \text { Angular mode order }
\end{aligned}
$$

For each layer labeled by 'j' the field components $E_{Z}^{j}$ and $B_{Z}^{j}$ must obey the reduced wave Eq. 2.

$$
\begin{aligned}
& {\left[\frac{\partial^{2}}{\partial r^{2}}+\frac{1}{r} \frac{\partial}{\partial r}+\left(k_{0}^{2} K_{j}^{2}-\frac{v^{2}}{r^{2}}\right)\right] B_{Z}^{j}(r)=0} \\
& {\left[\frac{\partial^{2}}{\partial r^{2}}+\frac{1}{r} \frac{\partial}{\partial r}+\left(k_{0}^{2} K_{j}^{2}-\frac{v^{2}}{r^{2}}\right)\right] E_{Z}^{j}(r)=0}
\end{aligned}
$$

Clearly, Eq. 2 form Bessel-equations whose analytical solutions are well known (Abramowitz and Stegun 1984).

For the fiber core ('co': $\mathrm{j}=0$ ) and the fiber cladding ('cl': $\mathrm{j}=\mathrm{p}+1$ ) these solutions can be expressed as follow:

$$
\begin{gathered}
E_{Z}^{(r)}= \begin{cases}E_{Z}^{c o}\left(r_{0}\right) \frac{J_{v}\left(u^{c o}\right)}{J_{v}\left(u_{0}^{c o}\right)} & \text { for } r<r_{0} \\
E_{Z}^{c l}\left(r_{p}\right) \frac{H_{v}^{(1)}\left(u^{c l}\right)}{H_{v}^{(1)}\left(u_{p}^{c l}\right)} & \text { for } r>r_{p}\end{cases} \\
B_{Z}(r)= \begin{cases}B_{Z}^{c o}\left(r_{0}\right) \frac{J_{v}\left(u^{c o}\right)}{J_{v}\left(u_{0}^{c o}\right)} & \text { for } r<r_{0} \\
B_{Z}^{c l}\left(r_{p}\right) \frac{H_{v}^{(1)}\left(u^{c l}\right)}{H_{v}^{(1)}\left(u_{p}^{c l}\right)} & \text { for } r>r_{p}\end{cases} \\
u^{c o}=k_{0} K_{c o} r ; \quad u_{0}^{c o}=k_{0} K_{c o} r_{0} ; \quad u^{c l}=k_{0} K_{c l} r ; \quad u_{p}^{c l}=k_{0} K_{c l} r_{p}
\end{gathered}
$$

The parameters $E_{Z}^{c o}\left(r_{0}\right), E_{Z}^{c l}\left(r_{p}\right), B_{Z}^{c o}\left(r_{0}\right), B_{Z}^{c l}\left(r_{p}\right)$ are integration constants defining the longitudinal electric and magnetic field components at the interface ' $r=r_{0}$ : core to first layer' and at the interface ' $\mathrm{r}=\mathrm{r}_{p}$; last layer to cladding'.

The symbols ' $J_{v}$ ' and ' $H_{v}^{(1)}$, denote Bessel functions and Hankel functions of the first kind, respectively, (Abramowitz and Stegun 1984). In many text books, for example in Bures (2009), the solution of the wave equation for the cladding is expressed by the Bessel function ' $K_{v}$ ', which is sufficient for bound modes with the real valued propagation constants. But 
for the general cases of radiation and absorption the more general Hankel ' $H_{v}^{(1)}$, is required, which allow the mode fields to decline as well as increase in the cladding region.

The analytical solution of the wave Eq. 2 inside a homogenous cylindrical layer ' $\mathrm{j}$ ' is:

$$
\begin{aligned}
& E_{Z}^{j}(r)= {\left[A^{j} H_{v}^{(1)}\left(u^{j}\right)+B^{j} H_{v}^{(2)}\left(u^{j}\right)\right] } \\
& B_{Z}^{j}(r)= {\left[C^{j} H_{v}^{(1)}\left(u^{j}\right)+D^{j} H_{v}^{(2)}\left(u^{j}\right)\right] } \\
& \text { with } u^{j}=k_{0} K_{j} r
\end{aligned}
$$

The parameters $A^{j}, B^{j}, C^{j}, D^{j}$; are integration constants too while $H_{v}^{(1)}\left(u^{j}\right), H_{v}^{(2)}\left(u^{j}\right)$ denote Hankel functions of the first and second kind, respectively. All Bessel functions as well as the all Hankel functions can be calculated numerically based on simple algorithms which can be found in Abramowitz and Stegun (1984). For large radius ' $r$ ' the cylindrical multilayer system behaves like the planar multilayer system. In this case, the Hankel functions of the first ' $H_{v}^{(1)}$ ' and the second ' $H_{v}^{(2)}$ ' kind can be approximated by ' $\exp \left(\mathrm{i} \mathrm{k}_{0} \mathrm{~K}_{\mathrm{j}} \mathrm{r}\right)$ ' and ' $\exp \left(-\mathrm{i} \mathrm{k}_{0} \mathrm{~K}_{\mathrm{j}} \mathrm{r}\right)$ ', respectively. These exponential functions represent forward and backward running waves inside a planar layer system.

So far we ended up with analytical set of field solutions for the longitudinal field components within each individual homogeneous cylindrical layer. This set of solutions contains a bunch of integration constants to be determined as follows.

The integration constants $A^{j}, B^{j}, C^{j}, D^{j}$ can be determined by means of Maxwell's continuity requirements, see Eq. 5 .

$$
\begin{array}{ll}
E_{\varphi}^{j+1}\left(r_{j}\right) & =E_{\varphi}^{j}\left(r_{j}\right) ; B_{\varphi}^{j+1}\left(r_{j}\right)=B_{\varphi}^{j}\left(r_{j}\right) ; \\
\varepsilon_{j+1} E_{r}^{j+1}\left(r_{j}\right) & =\varepsilon_{j} E_{r}^{j}\left(r_{j}\right) ; B_{r}^{j+1}\left(r_{j}\right)=B_{r}^{j}\left(r_{j}\right) ; \\
E_{Z}^{j+1}\left(r_{j}\right) & =E_{Z}^{j}\left(r_{j}\right) ; B_{Z}^{j+1}\left(r_{j}\right)=B_{Z}^{j}\left(r_{j}\right) ;
\end{array}
$$

Equations (5) interconnect the field solutions at an interface $r=r_{j}$ between two adjacent cylindrical layers labeled by " $\mathrm{j}$ ” and " $\mathrm{j}+1$ ”. For the longitudinal (z-) field components Eq. 5 give rise to Eq. 6:

$$
\begin{aligned}
& {\left[\frac{\partial}{\partial r} E_{z}^{j+1}\left(r_{j}\right)\right]=\frac{K_{j+1}^{2}}{k_{0}^{2} \varepsilon_{j+1}}\left\{\frac{k_{0}^{2} \varepsilon_{j}}{K_{j}^{2}}\left[\frac{\partial}{\partial r} E_{z}^{j}\left(r_{j}\right)\right]+i \nu \frac{k_{0} \beta}{r_{j}}\left[\frac{1}{K_{j}^{2}}-\frac{1}{K_{j+1}^{2}}\right] B_{z}^{j}\left(r_{j}\right)\right\},} \\
& {\left[\frac{\partial}{\partial r} B_{z}^{j+1}\left(r_{j}\right)\right]=K_{j+1}^{2}\left\{\frac{1}{K_{j}^{2}}\left[\frac{\partial}{\partial r} B_{z}^{j}\left(r_{j}\right)\right]+i v \frac{k_{0} \beta}{r_{j}}\left[\frac{1}{K_{j+1}^{2}}-\frac{1}{K_{j}^{2}}\right] E_{z}^{j}\left(r_{j}\right)\right\}}
\end{aligned}
$$

Tracing the longitudinal (z-) field components through the boundaries permits the elimination of all integration constants $A^{j}, B^{j}, C^{j}, D^{j}$. Thus the remaining task will be the determination of the integration constants $E_{Z}^{c o}\left(r_{0}\right), E_{Z}^{c l}\left(r_{p}\right), B_{Z}^{c o}\left(r_{0}\right), B_{Z}^{c l}\left(r_{p}\right)$ denoting the field values at the innermost and outermost cylindrical boundary of the total waveguide.

A closer look to Eq. 6 reveals that the tracing procedure leads to a mutual coupling of electric and magnetic field components reflecting the strongly vectorial character of the fiber modes. The both field components are decoupled for $v=0$ only, and for small differences in the dielectric function of different layers leading to the well known scalar approximation for the weakly guiding fiber.

The whole tracing procedure is depicted in Fig. 2. The algorithm can be considered as a generalized transfer matrix formalism for cylindrical geometries.

By means of our algorithm the determination of the remaining integration constants has been reduced to a matching procedure of the cladding field ansatz according to Eq. 3 with the internal field obtained from tracing up from the fiber core to the outermost waveguide 
Fig. 2 Tracing algorithm for the longitunal filed components between two interfaces ( $r_{p}$ and $\mathrm{r}_{\mathrm{p}+1}$, respectively) of the fiber structure. Note: (1) Coupling of longitudinal electric and magnetic field components along the interface between different layers. (2) Decoupled longitunal electric and magnetic field components inside each homogeneous cylindrical layer

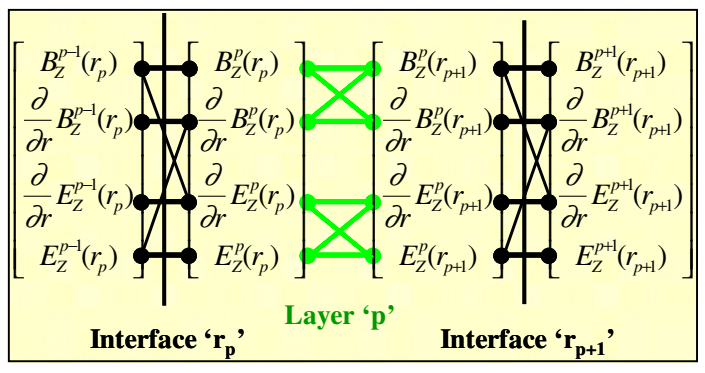

boundary. The matching procedure leads to multi parameter minimization problem that can be treated by means of a constraint optimization algorithm (Danzig 1963; Luenberger 1984).

To this end Eq. 2 can be rewritten for the outermost interface as summarized in Eq. 7.

$$
\begin{aligned}
& f_{1}=E_{\varphi}^{c l}\left(r_{p}\right)-E_{\varphi}^{p}\left(r_{p}\right) ; f_{2}=B_{\varphi}^{c l}\left(r_{p}\right)-B_{\varphi}^{p}\left(r_{p}\right) ; \\
& f_{3}=\varepsilon_{c l} E_{r}^{c l}\left(r_{p}\right)-\varepsilon_{p} E_{r}^{p}\left(r_{p}\right) ; f_{4}=B_{r}^{c l}\left(r_{p}\right)=B_{r}^{p}\left(r_{p}\right) ; \\
& f_{5}=E_{Z}^{c l}\left(r_{p}\right)-E_{Z}^{p}\left(r_{p}\right) ; \quad f_{6}=B_{Z}^{c l}\left(r_{p}\right)-B_{Z}^{p}\left(r_{p}\right) ;
\end{aligned}
$$

with $f=\sqrt{\left|f_{1}\right|^{2}+\left|f_{2}\right|^{2}+\left|f_{3}\right|^{2}+\left|f_{4}\right|^{2}+\left|f_{5}\right|^{2}+\left|f_{6}\right|^{2}}=0$

where the global minimum of ' $f$ ' has to be found numerically. For the final global minimum will provide the propagation constants of the modes supported by the given cylindrical layered waveguide, the relation " $f=0$ " may be regarded as the generalized dispersion relation of the waveguide structure under consideration.

The constraints will optimize the search path strategy. To this end, the boundary condition for the transverse electric and magnetic fields components at the outermost interface (see Eq. 3) were used to derive two complex equations allowing the determination of four real mode parameters, i.e. the complex-valued propagation constant ' $\beta$ ' and the ratio between the longitunal electrical and magnetic field components at the first interface.

$$
\begin{aligned}
& g_{1}=\left[\frac{\partial}{\partial r} E_{Z}^{c l}\left(r_{p}\right)\right]-\frac{\frac{\partial}{\partial r}\left[H_{v}^{(1)}\left(u_{p}^{c l}\right)\right]}{H_{v}^{(1)}\left(u_{p}^{c l}\right)} E_{Z}^{c l}\left(r_{p}\right)=0 ; \\
& g_{2}=\left[\frac{\partial}{\partial r} B_{Z}^{c l}\left(r_{p}\right)\right]-\frac{\frac{\partial}{\partial r}\left[H_{v}^{(1)}\left(u_{p}^{c l}\right)\right]}{H_{v}^{(1)}\left(u_{p}^{c l}\right)} B_{Z}^{c l}\left(r_{p}\right)=0 ;
\end{aligned}
$$

These two equations can be used as constraints avoiding local minima and providing a better convergence of our numerical algorithm. This leads to a classical nonlinear constraints optimization problem which can be formulated as follows: Minimize ' $f$ ', subject to $g_{1}<10^{-7}$ and $\mathrm{g}_{2}<10^{-7}$. Software packages like MATLAB provide appropriate tools for handling the optimization problem. Exactly at the cut-off, the concept of constraints optimization in general is still valid, but separate equations for the dispersion relation (8) have to be derived using the special case where the wave vector for the cladding region is approaching zero. Otherwise a singularity is occurring and no solution can be found. 


\section{Algorithm summary}

To summarize, the newly proposed algorithm includes the following steps: 1 . Decompose the given structure into a set of homogeneous cylindrical layers, bounded by a core and a cladding region; 2 . Derive and solve the cylindrical wave equation in the core and cladding region analytically and for all fiber layers analytically or numerically; 3 . Apply boundary conditions to connect the field components on both sides of the interface. Be advised about the mutual coupling of electric and magnetic field components at the interface (Okoshi 1982); 4. Formulate a modified dispersion relation to be minimized under the constraints for electric and magnetic fields imposed by the continuity conditions; 6 . Solve the resulting set of algebraic equations using Constraints Optimization methods as a function of the complex propagation constant and the complex ratio of the electric and magnetic fields a the first interface.

The whole algorithm has been implemented into the fiber module of the software package ATSOS which is available form www.doos.de (ATSOS 2008).

\section{Case examples}

After discussing the basic principle of modal field calculations in multilayer cylindrical waveguide structures, we will discuss three specific fiber configurations. With these examples, we will demonstrate the typical transitions from anti-resonant to resonant operational regime in the dispersion chart for the complex propagation constant of an ARROW fiber, the propagation characterristics of short and long range plasmon in a metal coated fiber, and the evanescent coupling between the core and an outside ring region inside a single fiber.

\subsection{ARROW fibers}

ARROW fibers concentrate the guided optical field in areas with lower refractive indices than their adjacent media by properly tuned interference effects.

We consider a hollow core ARROW fiber with an air-filled core (core diameter $150 \mu \mathrm{m}$ ) surrounded by high refractive index $(n=1.45)$ cylindrical wall of $85 \mu \mathrm{m}$ thickness. The light guiding properties of the structure depend strongly on the wall thickness and wavelength.

As a consequence the fiber modes radiate to a very small or to large amount into the surrounding air as a function of wavelength. These leaky mode characteristics are depicted in Fig. 3.
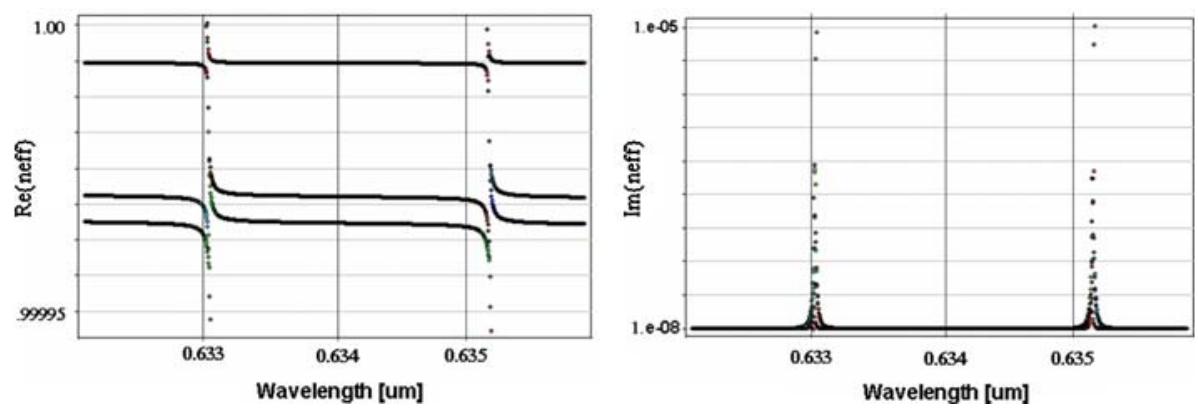

Fig. 3 Real (left) and imaginary (left) part of the complex dispersion relation for an air core ARROW fiber 

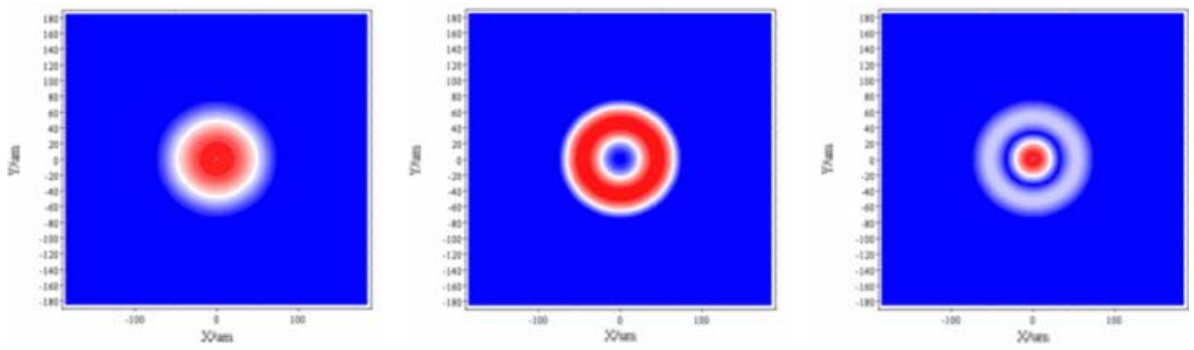

Fig. 4 Leaky mode intensity profiles at $634 \mathrm{~nm}$ for the $\mathrm{HE}_{11}$ mode (left), the $\mathrm{EH}_{11}$ mode (middle), and the $\mathrm{HE}_{21}$ mode (right)

The field intensity profiles for the first three modes at $634 \mathrm{~nm}$ are shown in Fig. 4.

As depicted in Fig. 3, the leakage of the modes is extremely strong around 633 and $635.1 \mathrm{~nm}$ and will continue at periodically spaced locations on the wavelength axis. This behaviour is typically for air-core ARROW-fibers whose spectral response consists of many narrow resonant changes between high and low radiation losses. The periodicity as well the desired wavelengths can be designed, selecting the appropriate thickness of the cylindrical wall by means of the presented algorithm. A majority of filtering applications requires introducing some kind of a resonant structure inside the fiber core. Therefore, using an ARROW fiber design no additional resonant structure for a filter application is required.

\subsection{Fiber surface plasmons}

In recent years the interest in the detection of thin ad-layers or single molecules attached to the surface of a metal layer has increased (Jacobsen et al. 2002). For good detection efficiency the appropriate mode excitation with a strong field enhancement at the outside metal interface has to be selected.

To give an idea about the design of such an application, the dispersion characteristic of a metal cladded fiber will be analyzed more detailed. The fiber consists of a quartz core (diameter: $0.001-1.1 \mu \mathrm{m}, n=1.457)$ coated by a $50 \mathrm{~nm}$ thick gold layer $(n=0.19+j 3.5)$. The ambient medium is air. Material properties were taken from Palik (1985). The wavelength is set to $633 \mathrm{~nm}$.

This fiber can support surface plasmons at angular order zero (TM modes) and hybrid modes of the types $\mathrm{EH}_{\mathrm{NM}}$ and $H E_{\mathrm{NM}}$. Hybrid modes couple more or less strongly to surface plasmons where the field enhancement is fundamentally different depending on the mode characteristics. Calculating the complex dispersion chart for a radius variation of the core from 1 to $1100 \mathrm{~nm}$ gives the characteristic curves of the metal cladded fiber structure (Fig. 5).

The strong field enhancement at the metal-cladding interface provides high potential for sensor applications. The right mode ' $\mathrm{EH}_{21}$ ' in Fig. 6 shows the highest field enhancement on the fiber outside. To excite the mode with the desired characteristic a tapered fiber structure can be used (Daxhelt et al. 2005). The presented approach allows the exact calculation of the complex dispersion relation for metal cladded fiber taken strong absorption of the metals into account.

\subsection{Core to ring coupling inside a multi-layer fiber}

The coupling process requires that two wave guiding systems are sufficiently close to achieve an overlap of their modal fields. The power transfer between the systems is determined by 

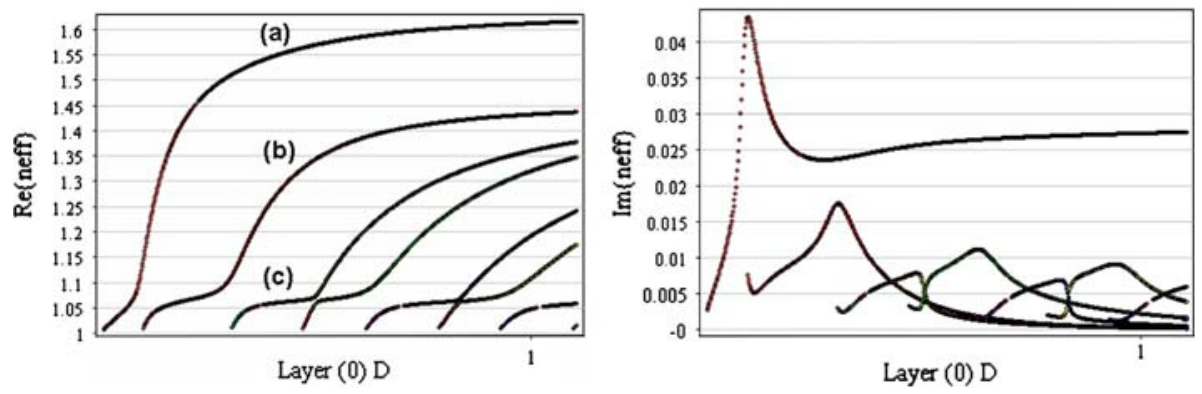

Fig. 5 Dispersion relation for the real part of 'neff' (left) and the imaginary part of 'neff' (right) as a function of fiber core thickness $(1-1,100 \mathrm{~nm})$
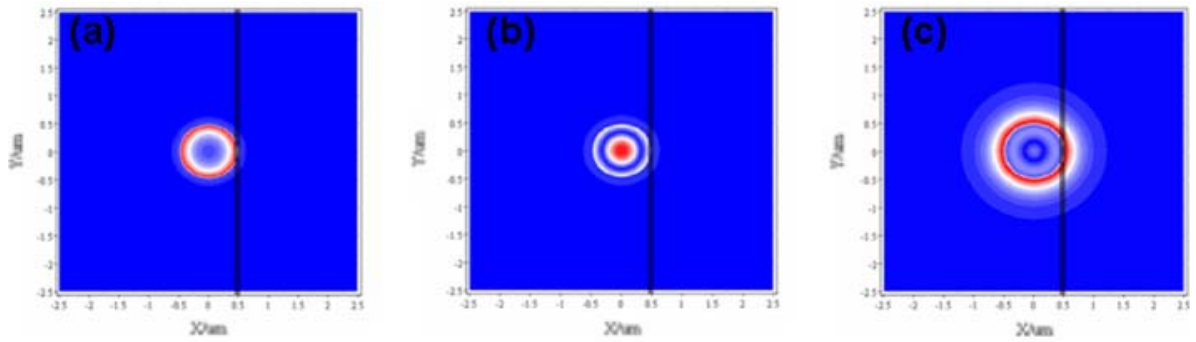

Fig. 6 Field intensity profiles for different hybrid fiber modes: $\mathbf{a} \mathrm{EH}_{11}$ shows large field enhancement at the core-metal interface; $\mathbf{b} \mathrm{HE}_{11}$ shows no field enhancement at the metal layer; $\mathbf{c} \mathrm{EH}_{21}$ shows the typical character of a coupled fiber-plasmon mode with large field enhancement at the outside metal-cladding interface

the difference in the propagation constants of the modes. In planar coupler geometries where the refractive index and the thickness of both waveguides are identical, the modes are identical and phase matched for all wavelengths. For asymmetric coupling the phase matched condition is given only at a discrete wavelength. This wavelength is determined by the intersection point of the mode dispersion of the isolated systems. At the wavelength characterized by intersection points a complete power exchange between the coupled systems is possible, whereas away from this wavelength the power transfer decreases to very small values resulting in a highly wavelength selective coupling device. The degree of the wavelength selectivity depends on the difference regarding the slopes of the dispersion curves at the intersection points (Chung and Safaai-Jazi 1992). In general, dissimilar systems are much more wavelength selective than similar systems. Such systems are attractive as narrow-band spectral filters. We will show that in a core to ring coupling schema inside a single fiber the wavelength selective characteristics of the coupling is always present. The same approach as known from the planar structures is used. First, the isolated systems are investigated and then combined as shown in Fig. 7. First, the dispersion relations of the isolated systems are investigated. From the previous work (Iliew et al. 2004) there are already some hints that the dispersion characteristics of core and ring modes are slightly different.

Figure 8 shows that the dispersion of the core mode is steeper than for the ring modes. For a given wavelength, in our case $633 \mathrm{~nm}$, there is only one thickness where coupling occurs for equal core and ring thicknesses. For all other cases with equal propagation constant ' $\beta$ ' or 'neff' the core and ring thicknesses are different. 


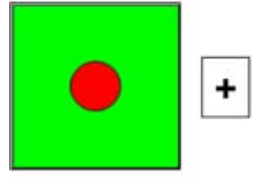

Core Mode

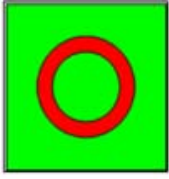

Ring Mode

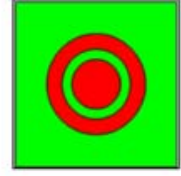

Super Modes

Fig. 7 Coupling takes place in the combined system if core and ring modes of the isolated systems intersect. Refractive index of ring and core $n=1.47$ (dark grey) and for the other area $n=1.45$ (light grey)

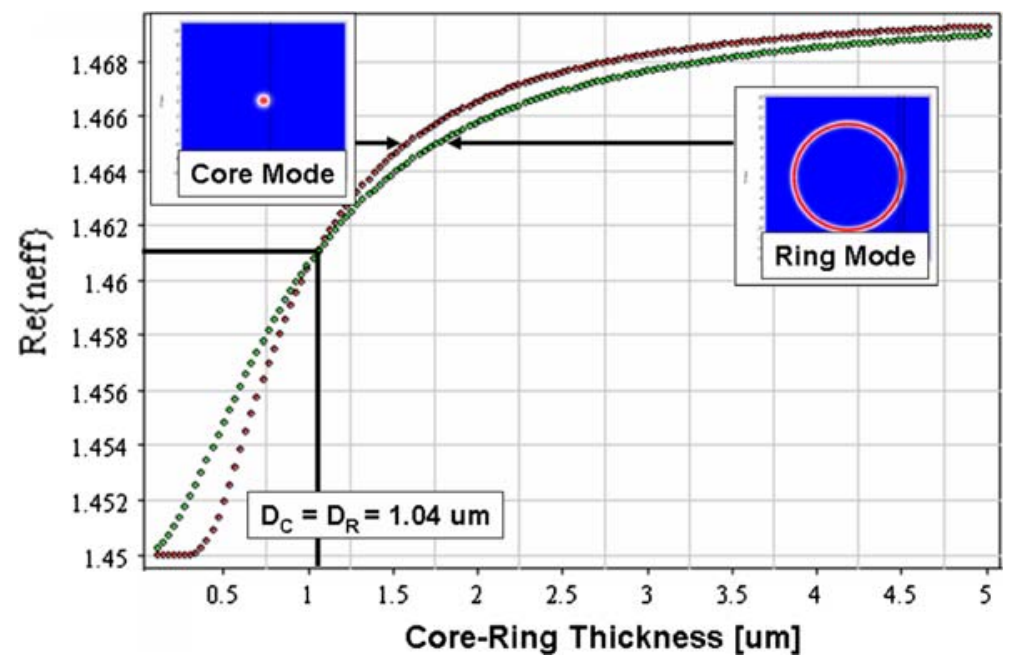

Fig. 8 Mode dispersion for isolated core and ring systems as a function of thickness (Wavelength $633 \mathrm{~nm}$ )

From this view point the coupling situation in the fiber is always wavelength selective and fundamental different from the planar case. The wavelength selective coupling can be demonstrated using the dispersion curves of the isolated and the combined systems as the function of wavelength shown in Fig.9.

The ring to core coupling can convert a small Gaussian beam into a large annular intensity distribution for selected wavelengths and with a flexible coupling length depending on the core-ring distance.

\subsection{Summary and future steps}

The application range of the proposed constraints optimization covers dielectric or metallic multi-layer stacks, optical waveguides, multi-layer- and hollow-core fibers, Plasmon excitation, leaky mode determination, coupling phenomena. The extension of the mode propagation to radiation and absorption phenomena requires a complex valued propagation constant and the replacement of the Bessel function ' $K_{v}$ ' with the more general Hankel function of the first kind in the cladding region. It is well known that the transition from guided modes to radiation modes is a challenging problem for any numerical algorithm. The here presented concept of a rigorous mode solver for multilayer fiber configurations using a Constraint Optimization method is no exemption. Nevertheless our method was numerically stable, modeling the transition from the so-called ARROW fiber modes to guided fiber modes. Avoiding the exact 


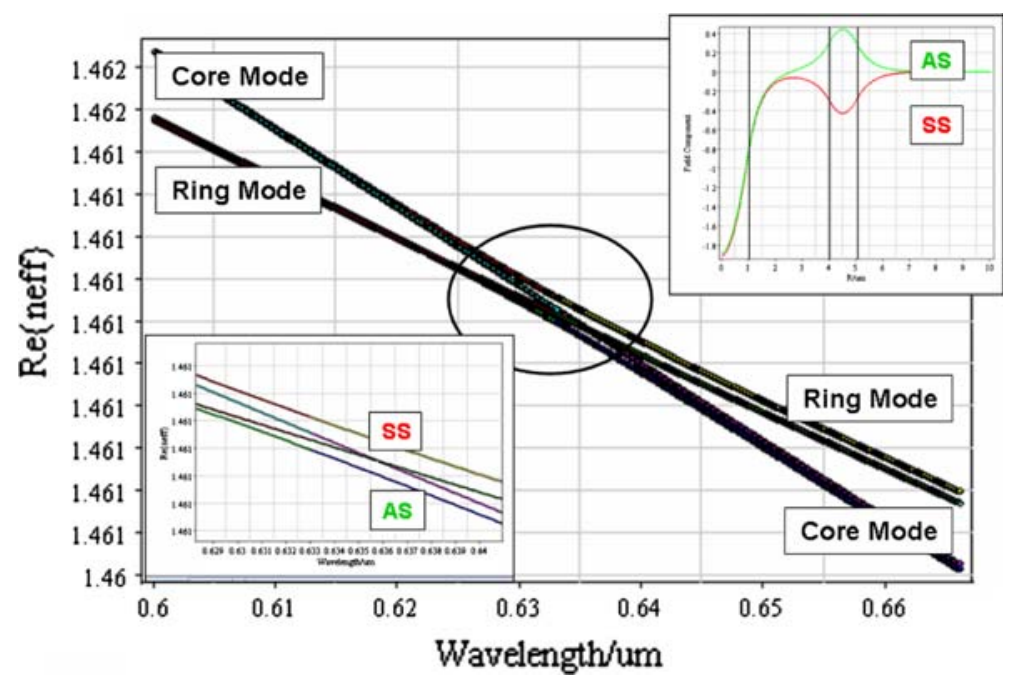

Fig. 9 Dispersion relation of isolated core $\left(D_{C}=1.04 \mathrm{um}\right)$ and ring $\left(D_{R}=1.04 \mathrm{um}\right)$ system and dispersion relation of the combined structures with intersection and supermodes splitting around the coupling wavelength of $633 \mathrm{~nm}$ (Zoom lower left corner). Field profiles of Symmetric Supermode (SS) and Asymmetric Supermode (AS) (upper right corner) are depicted

cut-off is recommended. Unfortunately, trying the same transition in the other direction from guided fiber modes to ARROW fiber modes proved to be difficult even with the application of a mode tracing algorithm.

The presented concept can be extended easily to nonlinear materials using a Runge-Kutta procedure to solve the wave equations inside the fiber layers. Additional applications of our rigorous fiber mode solver are in the areas of coaxial fibers and Bragg fibers.

\section{References}

Abramowitz, M., Stegun, I. (eds.): Pocketbook of Mathematical Functions. Verlag Harri Deutsch, Thun (1984) ATSOS (Analysis tool for stratified optical systems): Available from DOOS—Design \& optimization of optical systems. Tabarz, Germany. www.doos.de (2008)

Boerner, M., Trommer, G.: Lichtwellenleiter. Teubner Stuttgart (1989)

Born, M., Wolf, E.: Principles of Optics. Pergamon Press, London (1959)

Bures, J.: Guided Optics. Wiley, New York (2009)

Chilwell, J. et al.: Thin-films field-transfer matrix theory of planar multilayer waveguides and reflection from prism-loaded waveguides. JOSA A. 1, 742-753 (1984)

Chung, C.J., Safaai-Jazi, A.: Narrow-band spectral filter made of W-index and step-index fibers. J. Lightwave Technol. 10, 42-45 (1992)

Danzig, G.: Linear Programming and Extension. Princeton University Press, Princeton (1963)

Daxhelt, X., Martineau, L., Bures, J.: Influence of the fiber index profile on vectorial fiber modes and application to tapered fiber devices. J. Lightwave Technol. 23, 1874-1880 (2005)

Iliew, R., Etrich, C., Lederer, F.: Remote Coupling in Bragg fibers. Opt. Lett. 29, 1596-1598 (2004)

Jacobsen, V., Menges, B. et al.: In-situ thin film diagnostics using waveguide mode spectroscopy. Thin Solid Films 409, 185-193 (2002)

Luenberger, D.: Linear and Nonlinear Programming. Addison-Wesley, Reading (1984)

Okoshi, T.: Optical Fibers. Academic Press, New York (1982)

Palik, E.D.: Handbook of Optical Constants of Solids. Academic Press, New York (1985) 
Rowland, K.J., Afshar, S., Monro, T.M.: Bandgaps and antiresonances in integrated-ARROWs and Bragg fibers. Opt. Express 16, 17935-17951 (2008)

Yan, M., Shum, P.: Antiguiding in microstructured optical fibers. Opt. Express 12, 104-116 (2004)

Yeh, P., Yariv, A., Marom, E.: Theory of bragg fiber. J. Opt. Soc. Am. 68, 1196-1201 (1978) 